\title{
Biophysics across time and space
}

\section{Ewa K. Paluch}

MRC Laboratory for Molecular Cell Biology, University College London Institute for the Physics of Living Systems, University College London Physiology, Development and Neuroscience, University of Cambridge

e.paluch@ucl.ac.uk

Understanding the behaviour of almost any biological object is a fundamentally multiscale problem - a challenge that biophysicists has been increasingly embracing, building on two centuries of biophysical studies at a variety of length scales.

Until recently, biophysics was generally associated with studies of protein folding and ion channels. And with good reason: when the field underwent a tremendous expansion in the second half of the $20^{\text {th }}$ century, many of its key findings concerned the physical mechanisms behind molecular structures and assemblies. These successes were a boon for structural and molecular biology, but ultimately narrowed the focus of what was then a blossoming field. Now, research in biophysics is returning to the bigger picture spanning multiple length scales - proving that physics has just as much to say about the living as it does inanimate matter.

Historically, biophysics emerged between the $18^{\text {th }}$ and the $19^{\text {th }}$ centuries as part of the study of animal physiology. Luigi Galvani, who laid the foundations of electrophysiology by studying the effect of electricity on frog leg muscle movements in the late $18^{\text {th }}$ century, could be qualified as an early biophysicist. But the beginning of biophysics is usually attributed to a group of physiologists working around Johannes Müller in Berlin in the 1840s, who established physiology as an experiment-driven discipline founded in chemistry and physics. Their work went against the prevailing view that animals are governed by different laws than those describing inanimate objects ${ }^{1}$. Instead, they promoted 
the notion that living matter is, just like inanimate matter, governed by the laws of physics - a view revisited 70 years later by D'Arcy Thompson in the context of morphogenesis ${ }^{2}$.

Müller's circle, sometimes called the Berlin school of physiology, included scientists famous for their contributions to physics, such as Hermann von Helmholtz, and generated important insight into - among other things electrophysiology, the physics of muscles and nerves, and the physiology of vision. What can be considered the first biophysics textbook, discussing the physics of physiology, was published in 1856 by Adolf Fick under the title "Medical Physics".

The actual term biophysics is attributed to Karl Pearson, who in "the Grammar of Science" in 1892 noted that this branch of science was still underdeveloped, but "not improbably [had] an important future". The popularity of biophysics rose in the first half of the $20^{\text {th }}$ century. While neuro- and muscle physiology continued to develop ${ }^{1}$, early embryologists increasingly approached the question of morphogenesis, or acquisition of shape, in development as a question of mechanics ${ }^{3}$. In 1917, D’Arcy Thompson published his famous essay “On Growth and Form", in which he argued that physics must guide all studies of shape, growth and pattern formation in biology ${ }^{2}$. These developments led to the creation of departments dedicated to biophysics ${ }^{4}$ and culminated in the foundation of the Biophysical Society as an offspring of the American Physiological Society in 1957.

Over the course of the $20^{\text {th }}$ century, the focus of biophysics progressively shifted from the macroscale to the nanoscale. This transition was initiated by a growing interest in the physical basis of genes, famously discussed by the physicist Erwin Schrödinger in his essay "What is Life" 5 . The subsequent elucidation of DNA structure in 1953 by James Watson and Francis Crick, based on X-ray crystallography images by Rosalind Franklin ${ }^{6}$, is arguably the most famous advance of structural biophysics. It motivated innumerable studies of protein structures that, for a while, integrated biophysics within molecular biology. 
In the last couple decades, biophysics has expanded from largely reductionist approaches - at the scale of organisms or molecules - to the realm of what is often called systems biology. This evolution stemmed from a growing interest in the emergent properties of biological systems - cells, tissues, organisms displaying cooperative behaviours that cannot be directly extrapolated from the properties of their components. Molecular interactions at the sub-nanometre scale control molecular assemblies on a scale spanning tens of nanometres, which determine the properties of cells measuring tens of micrometres. Yet detailed knowledge of the underlying processes on one scale is not necessarily sufficient to understand behaviours on another.

This is evident in the fact that a cell's shape, mechanics, or signalling behaviour cannot be divined from nanoscale dynamics in the molecularly crowded environment of the cell surface (Refs Kuo and Diz-Munoz in this issue). Similarly, although tissue behaviour is controlled by cell dynamics and interactions, it cannot be directly predicted from cellular properties or from the molecular principles that control them (Ref Trepat in this issue). The same problem exists at a higher scale, in investigations of collective behaviour in groups of animals (Ref Gov in this issue).

Tools from statistical physics have been invaluable in providing a conceptual framework to understand emergent multiscale properties in biology (Refs Kuo, Trepat, Gov in this issue). Emergent behaviours in a complex system can often be described in the form of phase diagrams, as a function of variables describing specific properties of the system components (Ref Trepat in this issue). Such approaches are powerful because they help identify key mesoscale principles governing the collective behaviour. Transitions in behaviour — be it a cell's state, the shape of a tissue or the directional polarization of a group of ants - can then be described in the rich physical framework of phase transitions.

One particular biological complexity that is rarely addressed is the presence of multiple feedbacks between scales. The nanoscale organization of cytoskeletal 
networks determines cell mechanics and shape (reviewed in ${ }^{7}$ ), but the binding affinities of multiple cytoskeleton components are force sensitive. Some cytoskeletal cross-linkers form catch bonds, meaning their attachment is enhanced by mechanical load, while others form slip bonds that release under load. In this way, a cell's mechanics directly affects the organization of the cytoskeletal networks that govern it. At a more complex level, cell mechanics influences cellular signalling, with many cell types responding to, for example, the stiffness of their environment. Such mechanosensing behaviour also feeds back on cytoskeletal organization, and thus the resulting cellular mechanical properties. There is at present no theoretical framework that systematically includes such multiscale feedback loops - they are merely occasionally added ad hoc when specific feedback mechanisms are known.

As biophysics has undergone this transition from reductionist studies on one scale to multiscale approaches, there have been occasional attempts to recast it as 'physical biology' in attempt to clarify the distinction. Such semantic choices might become less relevant as the field expands into yet new directions. For instance, biophysicists have recently started exploring quantitative classifications of animal behaviour (Ref Brown in this issue). A predictive theory of behaviour has so far proven elusive, mostly because its connection to the genetic and physiological variables that control it is at best poorly understood. Although they do not provide a predictive framework, quantitative classifications based on dimensionality-reduction techniques are starting to unveil the behavioural state space, laying the ground for formal theory (Ref Brown in this issue).

Similar classifications using dimensionality reduction have been used extensively in recent years to describe changes in gene expression landscapes during cellular state changes ${ }^{8}$. There, statistical physics approaches are being developed using, for example, transcriptome entropy to describe cellular states 9 . How much predictive power such approaches will have to understand biological fate changes is an exciting question for future research. Going one step further, it remains to be seen whether statistical physics is the right framework to model 
the cross-talk between genetic networks, animal behaviour and evolution. It is also possible that novel biophysical approaches will be needed to address these highly complex questions that define living systems.

\section{Acknowledgements:}

EKP thanks Kevin Chalut for critical reading of the manuscript, and acknowledges funding from the Medical Research Council UK (MRC Programme Award MC_UU_12018/5) and the European Research Council (Starting Grant 311637-MorphoCorDiv).

\section{References}

1 Andersen, O. S. Introduction to Biophysics Week: What is Biophysics? Biophys J 110, E01-03 (2016).

2 Thompson, D. A. W. On Growth and Form. (Cambridge University Press, 1917).

3 Keller, R. Developmental biology. Physical biology returns to morphogenesis. Science 338, 201-203 (2012).

$4 \quad$ Hill, A. Why Biophysics? Science 124, 1233-1237 (1956).

$5 \quad$ Schrödinger, E. What is Life? , (Cambridge University Press, 1944).

6 Watson, J. D. \& Crick, F. H. Molecular structure of nucleic acids; a structure for deoxyribose nucleic acid. Nature 171, 737-738 (1953).

7 Koenderink, G. H. \& Paluch, E. K. Architecture shapes contractility in actomyosin networks. Curr Op Cell Biol 50, 79-85 (2018).

8 Stegle, O., Teichmann, S. A. \& Marioni, J. C. Computational and analytical challenges in single-cell transcriptomics. Nature reviews. Genetics 16, 133145 (2015).

9 Grun, D. et al. De Novo Prediction of Stem Cell Identity using Single-Cell Transcriptome Data. Cell stem cell 19, 266-277 (2016). 